\title{
Influences of club connectedness among young adults in Western Australian community-based sports clubs
}

\author{
Sharyn Burns ${ }^{*} \mathbb{D}$, Melissa Evans, Jonine Jancey, Linda Portsmouth and Bruce Maycock
}

\begin{abstract}
Background: Along with physical benefits, community-based sport provides opportunities to enhance connectedness, an important protective factor of social and emotional health. However, young Australians participating in sport have been found to drink alcohol at higher levels than their non-sporting peers, and many clubs serve unhealthy food and beverages. This study explored the association between the dependent variable, level of alcohol consumption (AUDITC) and connectedness to club and other health behaviours among young people aged 18-30 years who play club sport in Western Australia.
\end{abstract}

Methods: An online cross sectional survey measured levels of alcohol consumption (AUDIT-C), alcohol-related harm, connectedness (including volunteering and team cohesion), mental wellbeing, healthy food options and club sponsorship among young adults aged 18-30 years involved in sports clubs in Western Australia $(n=242)$.

Relationships and association between the dependent variable (AUDIT-C) and independent variables were assessed.

Results: Male sportspeople were more likely to drink alcohol at high-risk levels than females $(p<.001)$, and respondents belonging to a club that received alcohol-related sponsorship were more likely to drink at high-risk levels $(p=.019)$. Females were significantly more likely to want healthy food and beverage options provided at their clubs $(p=0.011)$. When all factors were considered team cohesion $(p=0.02)$, alcohol expectations $(p=<$ .001), occurrences of experienced alcohol-related harm $(p=<.001)$ and length of club membership $(p=0.18)$ were significant predictors of high-risk AUDIT-C $\left(R^{2}=.34\right.$, adjusted $\left.R^{2}=.33, F(4,156)=20.43, p=<.001\right)$. High-risk AUDIT-C and club connectedness predicted strong team cohesion $\left(R^{2}=.39\right.$, adjusted $R^{2}=.39, F(2,166)=53.74$, $p=<.001)$.

Conclusions: Findings from this study may inform policy and practice to enhance healthy behaviours among young adults participating in community sports clubs in Australia and other countries.

\section{Background}

In many countries, community-based sport is an important part of the lives of many young people [1-3]. In Australia, there are over 50,000 sport and active recreation clubs, with those involved taking on the roles of player, coach, committee member, spectator or volunteer [4].

\footnotetext{
*Correspondence: s.burns@curtin.edu.au

Collaboration for Evidence, Research and Impact in Public Health, School of Public Health, Curtin University, GPO Box U1987, Perth, Western Australia 6845, Australia
}

Sports involvement includes a range of individual and community-based benefits including connectedness [5].

Although benefits of sports club connectedness are discussed in some reports [6], few studies have measured associations between connectedness, alcohol consumption and other influences in the sports club setting. Connectedness provides a sense of belonging and it is believed having social ties to the community has links to positive outcomes such as positive mental health and health behaviour, less risk taking behaviour such as alcohol and

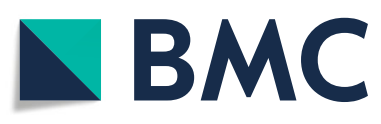

(c) The Author(s). 2020 Open Access This article is licensed under a Creative Commons Attribution 4.0 International License, which permits use, sharing, adaptation, distribution and reproduction in any medium or format, as long as you give appropriate credit to the original author(s) and the source, provide a link to the Creative Commons licence, and indicate if changes were made. The images or other third party material in this article are included in the article's Creative Commons licence, unless indicated otherwise in a credit line to the material. If material is not included in the article's Creative Commons licence and your intended use is not permitted by statutory regulation or exceeds the permitted use, you will need to obtain permission directly from the copyright holder. To view a copy of this licence, visit http://creativecommons.org/licenses/by/4.0/. The Creative Commons Public Domain Dedication waiver (http://creativecommons.org/publicdomain/zero/1.0/) applies to the data made available in this article, unless otherwise stated in a credit line to the data. 
other drug use, and better academic achievement for those in school [7-9]. Many club members play sport as well as volunteer with volunteers reporting higher levels of wellbeing, social connectedness, self-esteem and self-efficacy than non-volunteers [10].

However, people belonging to sporting clubs report higher levels of alcohol consumption compared to the general population [11]. In Australia [12, 13], and other countries, such as the US [14] and New Zealand [15], strong social and cultural norms are associated with alcohol consumption and sport.

Social alcohol consumption has been associated with team cohesion and bonding in team sports [16] with association between team social cohesion and risky alcohol consumption correlated to the frequency of team social events involving alcohol [17]. Despite these associations, players reporting positive team cohesion are more likely to continue participation [18]. There is also evidence to suggest involvement in sporting clubs provides young people protection from the uptake of risky drinking [19] due to the reduced levels of 'unsupervised leisure time' and boredom, resulting in less time and inclination for anti-social behaviour [20]. The social cohesion and support found in community sporting clubs may also act as a protective factor [12].

Club ethos and environment are important factors in attracting and retaining members. Clubs often aim to attract families making sports clubs an important community venue and social centre for all ages [12]. For many community-based sports clubs, the bar provides not only access to alcohol and revenue for the club, but a community hub, which fosters connectedness and socialisation $[13,21]$. However, the balance between alcohol access and responsible drinking is important. A study of 33 Australian community football clubs found having the bar open for more than 4 hours; serving intoxicated patrons; and alcohol promotions, to be associated with lower levels of perceived club safety and participation [13]. Provision of food contributes to club engagement and is a strategy of responsible alcohol consumption [11, 12], however, many sports clubs [22] and sporting events provide energydense and nutrient poor food and beverages [23] which are often supported by industry sponsorship [24]. Despite this there have been significant successes in reduction of risky alcohol consumption and related harm among Australian sports clubs when environmental and policy changes are implemented ( $n=43$ intervention; $n=45$ control community football clubs) [11].

Sports clubs play an important role in the sociocultural environment of many countries [23], with complex associations between club connectedness and health behaviours. There is little doubt as to the physical and mental health benefits of sports participation [25] or of the benefits of connectedness to mental and social wellbeing [7]. However, despite some success with structured interventions [11] the culture of risky alcohol consumption and community sports is of concern [26]. This study explored the association between the dependent variable, level of alcohol consumption (AUDIT-C) and connectedness to club and other health behaviours among young people aged 18-30 years who play club sport in Western Australia. This paper extends the literature around alcohol consumption and sports club participation to consider the influence of club connectedness.

\section{Method}

A cross sectional online survey including questions related to alcohol consumption, alcohol-related harm, healthy eating options, sponsorship, club connectedness (including volunteering) and mental wellbeing was administered. Ethics approval was received from the Curtin University Human Research Ethics Committee (HRE2016-0241). Interested participants clicked the survey link and were provided an online Participant Information Form describing the project. Participants were asked to indicate consent via an online question before they were able to proceed to the survey. Helplines and support services were provided for participants.

\section{Participants}

Young adults aged between 18 and 30 who live in Western Australia and participate in team sports were invited to participate in the online survey, which was open for a 2-month period. Recruitment occurred through convenience sampling using a dedicated project Facebook Page, club Facebook Pages and newsletters, other social media sites and snowball technique. Due to the convenience and snowball sampling methods employed, it is unknown how many clubs posted the survey information. A new Facebook business page was created for the study to enhance communication and recruitment, a strategy successfully employed in another alcohol study with young people [27]. Paid Facebook advertising assisted recruitment. An optional prize draw was offered as an incentive for participation. Efforts to ensure that duplicate survey completion was detected included investigation of similar or identical email addresses and IP addresses.

\section{Instrumentation \\ Demographics}

Demographic questions included age, gender (male, female, other), postcode, ethnicity, employment and student status. Age and location were restricted to ensure that only the intended target group completed the survey. 


\section{Involvement in sport}

Involvement in sport was determined by four questions that identified the main sport participants played during the last 12 months, the length of involvement and weekly time commitment.

\section{Availability of alcohol}

To determine availability of alcohol, participants were asked if their club had alcoholic drinks available for purchase, and how often the bar was open. Participants were also asked if food is served with alcohol. These questions were developed for this survey.

\section{Alcohol consumption}

The Alcohol Use Disorders Identification Test-Consumption (AUDIT-C) was employed to measure levels of alcohol consumption [28]. AUDIT and AUDIT-C are well validated and widely used tools developed for the World Health Organisation [28-30]. The AUDIT-C includes three consumption questions, is considered a sensitive indicator of alcohol consumption [31] and has been used with young sports people [32] and student samples [33]. Consistent with other research with young adults scores were collapsed into binary categories: low-risk $(<6)$ and high-risk $(\geq 6)$ consumption [34].

\section{Alcohol-related harm}

Experienced, witnessed and second-hand alcohol-related harms were measured using previously validated scales $[35,36]$. These questions were modified slightly to reflect consumption associated with teammates or sports club members during the last season. Harms as a result of other club members' alcohol consumption were measured using a 10-item scale (5-point Likert scale) [36, 37]. Witnessed harms included an eight-item scale on a 5-point Likert scale [35]. Experienced harms, harms associated with personal alcohol consumption, were measured using a validated version of the adapted Alcohol Problems Scale [36]. The 16-item scale included a 3point Likert scale. Alcohol expectancies were measured using the validated Brief Comprehensive Effects of Alcohol Scale (B-CEOA), a nine-item scale with responses agree, neither agree or disagree or disagree (score range 9-27) [38].

\section{Volunteering}

Involvement in volunteering was determined by questions of time committed, years of service and future planned volunteering. Volunteer motivation was measured via a validated scale (20 items; 7-point Likert) [39].

\section{Connectedness and cohesion}

Club connectedness was determined using a five-item Likert scale [40] previously used to measure connectedness [41]. Perceptions of fitting in with team and club members were measured using a validated four-item Likert scale [42].

Team cohesion was measured using an adapted version of the Group Environment Questionnaire (GEQ) (18 items) focusing on individual attraction and group integration [43]. The GEQ was analysed as a whole scale (team cohesion) and by the previously validated four constructs: Individual attractions to the Group-Task (an individual's feelings about personal involvement with the group task); Individual attractions to the Group-Social (individual's feelings about personal group social interactions); Group Integration Task (individual's perceptions around group unification of tasks); and Group Integration-Social (individual's perception of the group socially) [43]. The GEQ has previously been used to assess social connectedness and team cohesion in young people from sports clubs [44-46].

\section{Mental wellbeing}

Mental wellbeing was measured using the WarwickEdinburgh Mental Well-being Scale (WEMWBS) [47]. The 14 item scale has been used to measure the mental wellbeing of young adults who play sport $[48,49]$ and has demonstrated sound validity with a student sample (Cronbach alpha 0.89) [47].

\section{Food and drink availability}

To measure availability of food and beverages at their sports club, participants were asked if their club had a bar, canteen or vending machine and availability and frequency of use. Perception of the healthiness of the food and beverage options, and what they would like provided, was asked. Questions were adapted from an Australian survey targeting community sports clubs [50]. The following was included to clarify examples of food choices: "Examples of healthy food and beverages include fruit, salad rolls/sandwiches, unsalted nuts and water. Examples of unhealthy food and beverages soft drinks, energy drinks, pies, sausage rolls, hotdogs, hot chips, lollies, chocolate bars and crisps".

\section{Sponsorship}

Adapted questions were used to explore the presence and impact of sponsorship in community sporting clubs questions [51, 52]. These questions had previously been used to measure alcohol-sponsorship [51] and modified to measure non-alcohol sponsorship among Australian sportspeople [52]. Questions focused on sponsorship from alcohol-related or food and beverage industries and the type of sponsorship received. Participants were asked if their club/organisation/team received any sponsorship. Those who recalled sponsorship were provided a list of sponsors (for example, liquor store, brewery, hotel etc.) and then a question asking what type of 
sponsorship was received (for example, cash, uniforms, alcohol etc.). Separate questions were asked for alcohol and non-alcohol-related sponsorship.

\section{Data analysis}

Prior to conducting analyses total scores were calculated for scales and sub-scales. Nominated sports included Australian Rules Football League (AFL), hockey, netball, soccer, Ultimate Frisbee and Other. The 'other' group consisted of sports that had a low number of respondents and included American football, basketball, bouldering, cheerleading, cricket, floorball, gymnastics, judo, polocrosse, Quidditch, rowing, Rugby League, Rugby Union, softball, street roller hockey, swimming, taekwondo, tennis, volleyball and water polo.

The cross-sectional design of the survey allowed for the examination of relationships between variables [53]. Univariate analysis was used to determine the association between the dependent variable (AUDIT-C) and independent variables (sport, gender, age, location, student status, length of club membership, volunteer roles, alcohol-related sponsorship, non-alcohol-related sponsorship, experienced harm, second-hand harm, witnessed harm, club connectedness, team cohesion and mental wellbeing). Variables were compared between two levels of alcohol consumption (low- and high-risk drinkers) and gender (male and female). Chi square and Mann Whitney U and ANOVA tests were used to test relationships; Bonferroni correction was applied; Spearman's correlation measured association between variables. Dancey and Reidy's [54] interpretations guided the strength of correlations. Using these guidelines a correlation coefficient of +1 and -1 was considered perfect; + $0.9-+0.7$ and $-0.9--0.7$ as strong; $+0.6-+0.4$ and $0.6--0.4$ as moderate; $+0.3-+0.1$ and $-0.3--0.1$ as weak; and 0 as no correlation. Significance was determined at $p<0.001$ and moderate significance at $p<0.05$.

Multiple regression analysis explored associations between the dependent variable (AUDIT-C) and independent variables (see above). Due to data being nonnormally distributed non-parametric statistics were used to conduct the analyses. When analysing the data by gender, 'other', was excluded due to a low number of respondents for this option. Data were analysed using SPSS (version 25).

\section{Results}

Respondents $(n=242)$ were most likely to play Australian Football League (AFL), netball, hockey, soccer and Ultimate Frisbee; and to be female (approx. 60\%), with an average age of $26(S D=6.9)$. Almost half $(46 \%)$ of respondents studied at university full-time, and a third (32\%) had been a member of their club for more than 5 years. Not all respondents completed all questions (see tables).

Fifty-four per cent of respondents $(n=114 / 209)$ volunteered at their club, with $34 \%$ volunteering $2-3$ times a week or more. Overall, female respondents (57\%) volunteered more than males (50\%); however, males volunteered on a more frequent basis than females. Forty-six per cent of males volunteered 2-3 times a week, compared to $27 \%$ of females. The majority of respondents (64\%) reported average or above average mental wellbeing scores. Eighty-two percent of respondents reported a strong connection to their clubs, and $45 \%$ of respondents reported high team cohesion.

Overall, 60\% of respondents' clubs had a bar and a canteen $(n=106 / 177), 16.9 \%(n=30)$ a canteen only, and $3.4 \%(n=6)$ a bar only, with the canteen $(100 \%)$ and bar (87\%) most likely to be open on match days. For clubs with a bar most served meals (69\%) or snacks (16.8\%). Clubs received sponsorship from alcohol-related industry (26\%) and from other businesses (57.5\%) and food outlets (19\%).

A third (31\%) of respondents indicated they purchased food and beverages from their canteen once a week. Sixty seven percent of respondents indicated their club did not have a vending machine. Only $7 \%$ of respondents' clubs sold mostly healthy food and beverage options, and $62 \%$ said that they would like healthier options available.

\section{Alcohol consumption}

An AUDIT-C score was computed for 171 participants. Of these 32\% $(n=55)$ reported high-risk levels of alcohol consumption. Males were more likely to report high-risk levels of consumption compared to females (49\% vs. $21 \%$ respectively). Nearly two-thirds (64\%) of respondents $(n=168)$ had experienced one form of alcohol-related harm whilst drinking alcohol with their teammates or other sports club members, for example, having a hangover (56\%), feeling sick or vomiting (38\%), memory loss (20\%), and driving a car while under the influence of alcohol (12\%). At least one incident of alcohol-related harm had been witnessed by $40 \%$ of respondents and $43 \%$ had experienced second-hand harm as a result of teammates' drinking (Table 1).

Low and high-risk AUDIT-C scores were compared with key demographic variables. High-risk drinking was proportionally more likely to be reported by AFL players $(38.2 \%)(p=.020)$; males $(60.0 \%)(p=.001)$; respondents who were not students $(50.9 \%)(p=.014)$ and those belonging to a club that received alcohol-related sponsorship (38.9\%) $(p=.019)$. High-risk drinkers were significantly more likely to experience alcohol-related harm (M 4.0; SD 3.7) $(p<.001)$; have higher alcohol expectations (M 22.9; SD 3.4) $(p=.005)$; and report higher 
Table 1 Demographics and influencing factors for low and high-risk alcohol consumption

\begin{tabular}{|c|c|c|c|c|}
\hline & $\begin{array}{l}\text { Low-risk } \\
N(\%)\end{array}$ & $\begin{array}{l}\text { High-risk } \\
N(\%)\end{array}$ & $\begin{array}{l}\text { Total } \\
N(\%)\end{array}$ & $P$ value \\
\hline Sport & & & & $p=.020^{*}$ \\
\hline AFL & $19(16.4)$ & $21(38.2)$ & $40(23.4)$ & \\
\hline Hockey & $21(18.1)$ & $4(7.3)$ & $25(14.6)$ & \\
\hline Netball & $22(19)$ & $9(16.4)$ & $31(18.1)$ & \\
\hline Soccer & $13(11.2)$ & $4(7.3)$ & $17(9.9)$ & \\
\hline Ultimate Frisbee & $12(10.3)$ & $2(3.6)$ & $14(8.2)$ & \\
\hline Other*** & $29(25)$ & $15(27.3)$ & $44(25.7)$ & \\
\hline Total & 116 & 55 & 171 & \\
\hline Gender & & & & $p=.001^{*}$ \\
\hline Male & $34(29.3)$ & $33(60.0)$ & $67(39.2)$ & \\
\hline Female & $81(69.8)$ & $22(40.0)$ & $103(60.2)$ & \\
\hline Other & $1(0.9)$ & 0 & $1(.06)$ & \\
\hline Total & 116 & 55 & 171 & \\
\hline Age & & & & $p=.966$ \\
\hline $18-24$ years & $67(57.8)$ & $31(57.4)$ & $98(57.6)$ & \\
\hline $25-30$ years & $49(42.2)$ & $23(42.6)$ & $72(42.4)$ & \\
\hline Total & 116 & 54 & 170 & \\
\hline Location & & & & $p=.329$ \\
\hline Metro & $110(94.8)$ & $50(90.9)$ & 160 (93.6) & \\
\hline Regional/rural & $6(5.2)$ & $5(9.1)$ & $11(6.4)$ & \\
\hline Total & 116 & 55 & 171 & \\
\hline Student status & & & & $p=.014^{*}$ \\
\hline Non-student & $44(37.9)$ & $28(50.9)$ & $72(42.1)$ & \\
\hline University - full-time & $59(50.9)$ & $21(38.2)$ & $80(46.8)$ & \\
\hline University - part-time & $12(10.3)$ & $1(1.8)$ & $13(7.6)$ & \\
\hline TAFE - full-time & $0(0)$ & $1(1.8)$ & $1(0.6)$ & \\
\hline TAFE - part-time & $0(0)$ & $2(3.6)$ & $2(1.2)$ & \\
\hline Other & $1(0.9)$ & $2(3.6)$ & $3(1.8)$ & \\
\hline Total & 116 & 55 & 171 & \\
\hline Length of club membership & & & & $p=.303$ \\
\hline Less than 1 year & $26(22.4)$ & $6(10.9)$ & $32(18.7)$ & \\
\hline $1-2$ years & $30(25.9)$ & $15(27.3)$ & $45(26.3)$ & \\
\hline $3-5$ years & $28(24.1)$ & $14(25.5)$ & $42(24.6)$ & \\
\hline More than 5 years & $32(27.6)$ & $20(36.4)$ & $52(30.4)$ & \\
\hline Total & 116 & 55 & 171 & \\
\hline Volunteer roles & & & & $p=.437$ \\
\hline Yes & $58(50.0)$ & $31(56.4)$ & $89(52.0)$ & \\
\hline No & $58(50.0)$ & $24(43.6)$ & $82(48.0)$ & \\
\hline Total & 116 & 55 & 171 & \\
\hline Alcohol-related sponsorship & & & & $p=.019^{*}$ \\
\hline Yes & $22(19.5)$ & $21(38.9)$ & $43(25.7)$ & \\
\hline No & $68(60.2)$ & $22(40.7)$ & $90(53.9)$ & \\
\hline Unsure & $23(20.4)$ & $11(20.4)$ & $34(20.4)$ & \\
\hline
\end{tabular}


Table 1 Demographics and influencing factors for low and high-risk alcohol consumption (Continued)

\begin{tabular}{|c|c|c|c|c|}
\hline & $\begin{array}{l}\text { Low-risk } \\
N(\%)\end{array}$ & $\begin{array}{l}\text { High-risk } \\
N(\%)\end{array}$ & $\begin{array}{l}\text { Total } \\
N(\%)\end{array}$ & $P$ value \\
\hline Total & 113 & 54 & 167 & \\
\hline Non-alcohol related sponsorship & & & & $p=.229$ \\
\hline Yes & $60(53.1)$ & $36(66.7)$ & $96(57.5)$ & \\
\hline No & $21(18.6)$ & $6(11.1)$ & $27(16.2)$ & \\
\hline Unsure & $32(28.3)$ & $12(22.2)$ & $44(26.3)$ & \\
\hline Total & 113 & 54 & 167 & \\
\hline \multirow[t]{3}{*}{ Experienced harm } & M 1.9 & M 4.0 & M 2.6 & $p<.001^{* *}$ \\
\hline & SD 3.5 & SD 3.7 & SD 3.7 & \\
\hline & Cl 1.3-2.6 & Cl 2.9-5.0 & Cl $2.1-3.2$ & \\
\hline \multirow[t]{3}{*}{ Second-hand harm } & M 1.5 & M 1.39 & M 1.5 & $p=.199$ \\
\hline & SD 3.5 & SD 1.9 & SD 3.1 & \\
\hline & $\mathrm{Cl} 0.8-2.2$ & Cl $0.8-1.9$ & Cl 1.0-1.9 & \\
\hline \multirow[t]{3}{*}{ Witnessed harm } & M 1.9 & M 2.3 & M 2.1 & $p=.403$ \\
\hline & SD 4.4 & SD 3.8 & SD 4.2 & \\
\hline & Cl 1.2-2.8 & Cl 1.2-3.3 & Cl 1.4-2.7 & \\
\hline \multirow[t]{3}{*}{ Alcohol expectations } & M 20.7 & M 22.9 & M 21.4 & $p=.005^{*}$ \\
\hline & SD 4.6 & SD 3.4 & SD 4.4 & \\
\hline & Cl 19.8-21.6 & Cl 21.9-23.8 & Cl 20.7-22.1 & \\
\hline \multirow[t]{3}{*}{ Club connectedness } & M 21.4 & M 21.8 & M 21.5 & $p=.143$ \\
\hline & SD 3.1 & SD 3.4 & SD 3.1 & \\
\hline & Cl 20.8-21.9 & Cl 20.9-22.8 & Cl 21.1-21.9 & \\
\hline \multirow[t]{3}{*}{ Team cohesion (GEQ) } & M 117.7 & M 124.0 & M 118.6 & $p=.043^{*}$ \\
\hline & SD 21.8 & SD 24.8 & SD 23.1 & \\
\hline & Cl 113.5-121.9 & Cl 117.3-130.7 & Cl 115.3-121.9 & \\
\hline \multirow[t]{3}{*}{ Individual attractions to the Group-Social (ATG-S) } & M 32.6 & M 35.3 & M 33.5 & $p=0.034^{*}$ \\
\hline & SD 7.4 & SD 8.6 & SD 7.9 & \\
\hline & Cl 31.2-33.9 & Cl 32.9-37.7 & Cl 32.3-34.7 & \\
\hline \multirow[t]{3}{*}{ Individual attractions to the Group-Task (ATG-T) } & M 29.4 & M 29.8 & M 29.6 & $p=0.722$ \\
\hline & SD 5.32 & SD 6.9 & SD 5.9 & \\
\hline & Cl 28.5-30.4 & Cl 27.7-31.6 & Cl 28.7-30.4 & \\
\hline \multirow[t]{3}{*}{ Group Integration Task (GI-T) } & M 33.1 & M 34.1 & M 33.4 & $p=0.416$ \\
\hline & SD 7.3 & SD 7.0 & SD 7.2 & \\
\hline & Cl 31.7-34.4 & Cl 32.2-35.9 & Cl $32.3-34.5$ & \\
\hline \multirow[t]{3}{*}{ Group Integration-Social (GI-S) } & M 22.4 & M 24.9 & M 23.2 & $p=0.14$ \\
\hline & SD 6.1 & SD 6.4 & SD 6.3 & \\
\hline & Cl 21.2-23.5 & Cl 23.1-26.6 & Cl 22.2-24.11 & \\
\hline \multirow[t]{3}{*}{ Mental Wellbeing } & M 52.7 & M 53.1 & M 52.3 & $p=.782$ \\
\hline & SD 8.5 & SD 7.5 & SD 8.7 & \\
\hline & Cl 51.2-54.3 & Cl 50.9-55.1 & Cl 51.1-53.6 & \\
\hline
\end{tabular}

*. $p<0.05$ level

**. $p<0.01$ level

${ }^{* * *}$ Other = American football, basketball, bouldering, cheerleading, cricket, floorball, gymnastics, judo, polocrosse, Quidditch, rowing, Rugby League, Rugby Union, softball, street roller hockey, swimming, taekwondo, tennis, volleyball and water polo 
team cohesion (M 124.0; SD 24.8) ( $p=.043)$. There was no significant difference between low and high-risk drinking and age, location, length of club membership, volunteer roles, non-alcohol related sponsorship, second-hand harm, witnessed harm, club connectedness and mental wellbeing.

\section{Gender differences}

Males (45.7\%) were significantly more likely to play AFL while females were more likely to play netball $(32 \%)$ and hockey $(20.0 \%)(p<.001)$. While there was no significant difference between uptake of volunteer roles and gender, male respondents were significantly more likely to volunteer on a more frequent basis $(p=.037)$. Female respondents $(68.4 \%)$ were moderately significantly more likely to want more healthy food and beverage options at their club (53.4\%) $(p=.011)$. Male respondents were significantly more likely to report higher AUDIT-C scores (M 5.0; SD 2.3) $(p=.001)$, experience alcohol relatedharm (M 3.2; SD 3.4) $(p=.003)$, second-hand alcoholrelated harm (M 2.1; SD 3.9) $(p=.006)$, and witness alcohol-related (M 3.2; SD 5.3) $(p=.004)$. There was no significant difference between gender and length of club membership, alcohol expectations, club connectedness, team cohesion and mental wellbeing (Table 2).

\section{Multiple regression}

When all factors were considered, team cohesion $(p=$ $0.02)$, alcohol expectations $(p=<.001)$, occurrences of experienced alcohol-related harm $(p=<.001)$ and length of club membership ( $p=0.18)$ accounted for a significant $34.4 \%$ of the variability in AUDIT-C scores $\left(R^{2}=.34\right.$, adjusted $\left.R^{2}=.33, F(4,156)=20.43, p=<.001\right)$. In combination, club connectedness $(p<.001)$ and AUDIT-C $(p=.145)$ score accounted for a significant $39.3 \%$ of the variability in team cohesion $\left(R^{2}=.39\right.$, adjusted $R^{2}=.39, F$ $(2,166)=53.74, p=<.001)$.

\section{Correlations between variables}

AUDIT-C score was moderately significantly positively correlated with club connectedness $\left(r_{s}=.18, p<.05\right.$, twotailed, $N=171)$ and team cohesion $\left(r_{s}=.22, p<.01\right.$, twotailed, $N=171)$, however these correlations were weak. When team cohesion was collapsed to measure individual and group task and social constructs, moderately significant, but weak, positive correlations were found between AUDIT-C and Group Integrated-Social $\left(r_{s}=.22\right.$, $p<.01$, two-tailed, $N=171)$; Individual Attractions Group Social $\left(r_{s}=.25, p<.01\right.$, two-tailed, $\left.N=171\right)$; and Individual Attractions Group Task $\left(r_{s}=.16, p<.01\right.$, twotailed, $N=171)$. Moderate positive relationships were reported for AUDIT-C score with experienced harm $\left(r_{s}=\right.$ $.50, p<.001$, two-tailed, $N=168)$, alcohol expectations $\left(r_{s}=.38, \quad p<.001\right.$, two-tailed, $\left.N=167\right)$ and drinking alcohol with teammates $\left(r_{s}=.44, p<.001\right.$, two-tailed, $N=160$ ) (Table 3).

Club connectedness and team cohesion were moderately correlated $\left(r_{s}=.54, p<.001\right.$, two-tailed, $\left.N=190\right)$. Mental wellbeing score was significantly positively correlated with team cohesion $\left(r_{s}=.29, p<.001\right.$, two-tailed, $N=179)$ and club connectedness $\left(r_{s}=.28, p<.001\right.$, twotailed, $N=179$ ), however correlations were weak. All four constructs of team cohesion were also positively correlated with club connectedness and mental wellbeing.

All alcohol-related harms were significantly positively correlated, with most demonstrating moderate correlations. Experienced harm was significantly positively correlated with second-hand harm $\left(r_{s}=.32, p<.001\right.$, two-tailed, $N=168)$ and witnessed harm $\left(r_{s}=.42, p<.001\right.$, two-tailed, $N=168$ ), and second-hand harm and witnessed harm were significantly positively correlated $\left(r_{s}=.65, p<.001\right.$, two-tailed, $N=168$ ). Drinking alcohol with teammates was significantly positively correlated with experienced alcohol-related harm $\left(r_{s}=.45, p<.001\right.$, two-tailed, $N=$ 157), second-hand harm $\left(r_{s}=.20, p<.05\right.$, two-tailed, $N=$ $157)$ and witnessed harm $\left(r_{s}=.33, p<.001\right.$, two-tailed, $N=157$ ) (Table 3).

\section{Discussion}

Associations between club connectedness, alcohol consumption and other behaviours among young adults involved in community sports clubs were explored. Similar to other studies of sportspeople in Australia [52], New Zealand [55] and Ireland [56] males in this study reported to drink alcohol at riskier levels than females. These studies used the 10-item AUDIT as opposed to AUDIT-C to measure levels of alcohol consumption. Young Australian adult males are reported to consume alcohol at higher levels than females of the same age, as demonstrated within public [1] and university populations [35].

Strong team cohesion was a predictor of high-risk alcohol consumption. Team cohesion has been identified as a significant predictor of alcohol consumption amongst university sportspeople in the UK $(n=1785)$ [16], while teammates influence and the team environment has been found to influence higher levels of alcohol consumption amongst sports players [15, 49, 57]. However a study amongst female US college athletes $(n=174)$ found no significant association between team social cohesion (computed using an average of the Individual Attraction to Group-Social and Group Integration-Social scores) and hazardous drinking [17]. When team cohesion in this study was analysed using the four constructs; social, as opposed to task oriented constructs accounted for higher correlations to alcohol consumption, however although moderately significant these correlations were weak. 
Table 2 Demographics and influencing factors and gender (male and female)

\begin{tabular}{|c|c|c|c|c|}
\hline & $\begin{array}{l}\text { Male } \\
N(\%)\end{array}$ & $\begin{array}{l}\text { Female } \\
N(\%)\end{array}$ & $\begin{array}{l}\text { Total } \\
N(\%)\end{array}$ & $P$ value \\
\hline Sport & & & & $p<.001^{* *}$ \\
\hline AFL & $37(45.7)$ & $10(8.0)$ & $47(22.8)$ & \\
\hline Hockey & $3(3.7)$ & $25(20.0)$ & $28(13.6)$ & \\
\hline Netball & $0(0)$ & $40(32.0)$ & $40(19.4)$ & \\
\hline Soccer & $12(14.8)$ & $11(8.8)$ & $23(11.2)$ & \\
\hline Ultimate Frisbee & $4(4.9)$ & $12(9.6)$ & $15(7.8)$ & \\
\hline Other*** & $25(30.9)$ & $27(21.6)$ & $52(25.2)$ & \\
\hline Total & 81 & 125 & 206 & \\
\hline AUDIT-C category & & & & $p<.001^{* *}$ \\
\hline Low-risk & $34(50.7)$ & 81 (78.6) & $115(67.6)$ & \\
\hline High-risk & $33(49.3)$ & $22(21.4)$ & $55(32.4)$ & \\
\hline Total & 67 & 103 & 170 & \\
\hline Length of club membership & & & & $p=.761$ \\
\hline Less than 1 year & $14(17.3)$ & $24(19.0)$ & $38(18.4)$ & \\
\hline $1-2$ years & $22(27.2)$ & $33(26.2)$ & $55(26.6)$ & \\
\hline $3-5$ years & $22(27.2)$ & $27(21.4)$ & $49(23.7)$ & \\
\hline More than 5 years & $23(28.4)$ & $42(33.3)$ & $65(31.4)$ & \\
\hline Total & 81 & 126 & 207 & \\
\hline Volunteer roles & & & & $p=.357$ \\
\hline Yes & $41(50.6)$ & $72(57.1)$ & $113(54.6)$ & \\
\hline No & $40(49.4)$ & $54(42.9)$ & $94(45.4)$ & \\
\hline Total & 81 & 126 & 207 & \\
\hline Time spent volunteering & & & & $p=.037^{*}$ \\
\hline 2-3 times a week or more & $18(46.2)$ & $19(26.8)$ & 37 (33.6) & \\
\hline Once a week & $13(33.3)$ & $21(29.6)$ & $34(30.9)$ & \\
\hline Once a month or less & $8(20.5)$ & $31(43.7)$ & $39(35.5)$ & \\
\hline Total & 39 & 71 & 110 & \\
\hline Healthy food options & & & & $p=.011^{*}$ \\
\hline Yes, absolutely & $10(16.7)$ & $34(35.8)$ & $44(28.4)$ & \\
\hline Yes & $22(36.7)$ & $31(32.6)$ & $53(34.2)$ & \\
\hline No & $24(40.0)$ & $19(20.0)$ & $43(27.7)$ & \\
\hline Unsure & $4(6.7)$ & $11(11.6)$ & $15(9.7)$ & \\
\hline Total & 60 & 95 & 155 & \\
\hline \multirow[t]{3}{*}{ AUDIT-C score } & M 5.0 & M 3.9 & M 4.5 & $p=.001^{*}$ \\
\hline & SD 2.3 & SD 3.2 & SD 2.3 & \\
\hline & Cl 4.4-5.6 & Cl $3.5-4.3$ & Cl $4.0-4.7$ & \\
\hline \multirow[t]{3}{*}{ Experienced harm } & M 3.2 & M 2.3 & M 2.6 & $p=.003^{*}$ \\
\hline & SD 3.5 & SD 3.8 & SD 3.7 & \\
\hline & Cl 2.4-4.1 & Cl 1.5-3.0 & Cl 2.1-3.2 & \\
\hline \multirow[t]{3}{*}{ Second-hand harm } & M 2.3 & M 1.1 & M 1.5 & $p=.006^{*}$ \\
\hline & SD 3.9 & SD 2.4 & SD 3.1 & \\
\hline & Cl 1.2-3.1 & Cl 1.1-1.5 & Cl 1.1-1.9 & \\
\hline Witnessed harm & M 3.2 & M 1.4 & M 2.1 & $p=.004^{*}$ \\
\hline
\end{tabular}


Table 2 Demographics and influencing factors and gender (male and female) (Continued)

\begin{tabular}{|c|c|c|c|c|}
\hline & $\begin{array}{l}\text { Male } \\
N(\%)\end{array}$ & $\begin{array}{l}\text { Female } \\
N(\%)\end{array}$ & $\begin{array}{l}\text { Total } \\
N(\%)\end{array}$ & $P$ value \\
\hline & SD 5.3 & SD 3.2 & SD 4.2 & \\
\hline & $\mathrm{Cl} 1.9-4.5$ & $\mathrm{Cl} .7-1.9$ & Cl 1.4-2.7 & \\
\hline \multirow[t]{3}{*}{ Alcohol expectations } & M 22.1 & M 21.1 & M 21.5 & $p=.313$ \\
\hline & SD 3.7 & SD 4.7 & SD 4.4 & \\
\hline & Cl 21.2-23.0 & Cl 20.1-21.9 & Cl 20.8-22.1 & \\
\hline \multirow[t]{3}{*}{ Club connectedness } & M 21.7 & M 21.4 & M 21.5 & $p=.455$ \\
\hline & SD 3.3 & SD 3.0 & SD 3.1 & \\
\hline & Cl 20.9-22.5 & Cl 20.8-21.9 & Cl 21.1-21.9 & \\
\hline \multirow[t]{3}{*}{ Team cohesion (GEQ) } & M 119.2 & M 118.2 & M 118.6 & $p=.608$ \\
\hline & SD 25.6 & SD 21.6 & SD 23.2 & \\
\hline & Cl 113.2-125.3 & Cl 114.3-122.2 & Cl 115.3-121.9 & \\
\hline \multirow[t]{3}{*}{ Individual attractions to the Group-Social (ATG-S) } & M 34.3 & M 32.4 & M 33.1 & $p=0.308$ \\
\hline & SD 9.1 & SD 7.6 & SD 7.6 & \\
\hline & Cl 32.1-36.4 & Cl 30.9-33.8 & Cl 31.9-34.3 & \\
\hline \multirow[t]{3}{*}{ Individual attractions to the Group-Task (ATG-T) } & M 29.0 & M 29.1 & M 29.1 & $p=0.823$ \\
\hline & SD 6.9 & SD 5.9 & SD 6.3 & \\
\hline & Cl 287.4-30.6 & Cl 28.0-30.2 & Cl 28.2-40.0 & \\
\hline \multirow[t]{3}{*}{ Group Integration Task (GI-T) } & M 32.3 & M 33.8 & M 33.2 & $p=0.305$ \\
\hline & SD 7.6 & SD 6.9 & SD 7.2 & \\
\hline & Cl 30.5-34.0 & Cl 32.6-35.1 & Cl 32.2-34.3 & \\
\hline \multirow[t]{3}{*}{ Group Integration-Social (GI-S) } & M 23.7 & M 22.9 & M 23.2 & $p=0.14$ \\
\hline & SD 6.73 & SD 5.9 & SD 6.2 & \\
\hline & Cl 22.13-25.29 & Cl 21.9-24.1 & Cl 22.3-24.1 & \\
\hline \multirow[t]{3}{*}{ Mental Wellbeing } & M 54.2 & M 51.3 & M 52.4 & $p=.073$ \\
\hline & SD 8.0 & SD 9.0 & SD 8.8 & \\
\hline & Cl 52.3-56.1 & Cl 49.6-53.0 & Cl 51.1-53.7 & \\
\hline
\end{tabular}

*. $p<0.05$ level

**. $p<0.01$ level

***Other = American football, basketball, bouldering, cheerleading, cricket, floorball, gymnastics, judo, polocrosse, Quidditch, rowing, Rugby League, Rugby Union, softball, street roller hockey, swimming, taekwondo, tennis, volleyball and waterpolo

Club connectedness and AUDIT-C scores were also found to significantly correlate to team cohesion, particularly the social team cohesion constructs. To our knowledge, no studies have reported on the association between club connectedness and team cohesion. This is worthy of further exploration.

Respondent mental wellbeing was positively, although weakly, correlated with team cohesion and club connectedness, supporting previous findings $[16,49,58]$. The social nature of participating in team sport is believed to contribute to improved health and mental wellbeing [59], and research amongst US college students has revealed that connectedness acts as a protective factor against depression for athletes, which may be the result of having a social network and team support [60].

Volunteers are considered the most important resource for community sports clubs and provide opportunity to enhance club connectedness [61]. Approximately $65 \%$ of participants volunteered once a week or more. This is higher than the national findings of 39\% of adult Australians, and only 8.4 and $10.5 \%$ of young adults aged 18-24 and 25-34 years respectively, volunteering at least once a week with their club [62]. In contrast to other studies [62-64], a higher percentage of females volunteered for their club than males. However, these studies explored volunteering in the general public [64] or explored sport volunteer rates across the life span and did not specifically examine gender by age groups [62,63]. A negative correlation between the uptake of volunteer roles and length of club membership was found. Similarly, others have found similar associations, suggesting volunteers may give up volunteer roles if dissatisfied or perceive that they have fulfilled their obligations [65] . 


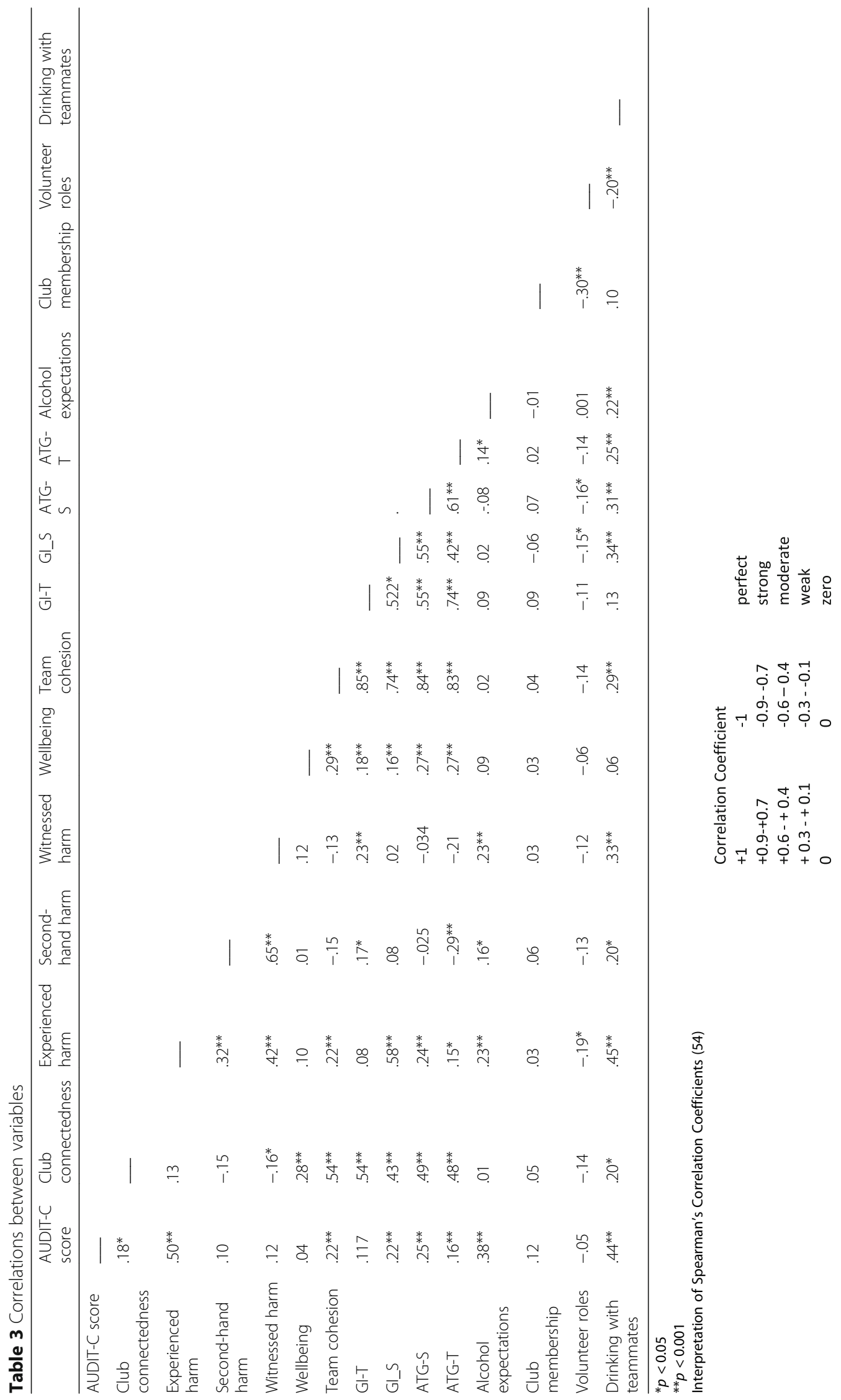


Females in this study were significantly more likely to want more healthy food options available at their club than males. While the promotion of food and beverages has been explored in some studies [66, 67], and another has explored stakeholders views [23]; there is limited research exploring sportspeople's attitudes towards the availability of healthy food in sports clubs. However, Australian female athletes are more opposed to unhealthy food advertising in sport than male athletes [68] and females have a stronger interest in healthy eating than males [69].

Participants were more likely to indicate that their team or club received non-alcohol related sponsorship (57.5\%) compared to alcohol-related sponsorship (25.7\%). It is unknown if recall of sponsorship is accurate, however another Australian study using similar measures found $31 \%(n=204)$ of sportspeople to report some sponsorship, with most $(95 \% ; n=194)$ receiving alcohol-related sponsorship. In contrast to the current study fewer $(n=26 ; 13 \%)$ received non-alcohol related sponsorship and $n=16$ (8\%) received both [52]. Respondents belonging to a club that received alcohol-related sponsorship were significantly more likely to consume alcohol at hazardous levels, supporting previous findings from Australia [52], New Zealand [51] and the UK [70] and in a systematic review [71]. Food and beverage sponsors, especially for energy-dense, nutrient-poor foods high-energy, do target sports venues and clubs [23]. More research to explore the impacts among young adults would be beneficial.

\section{Recommendations for intervention}

The findings of this study highlight the importance of sports clubs as community-based environments, which have the potential to enhance positive physical, social and emotional health outcomes [72, 73]. Some sports clubs have implemented strategies to create environments that recognise and capitalise on their role as community hubs [21]. The Good Sports program has demonstrated some positive changes to alcohol consumption especially among AFL and cricket clubs in Australia when policy-related strategies were implemented [11, 74]. After a 4 month intervention including behavioural and policy-related strategies an Irish controlled trial of sports clubs in two RCT found no reduction in alcohol consumption, or AUDIT scores, although there was reduction in the number of alcohol related harms reported [56].

A qualitative study $(n=22)$ found alcohol to be seen to help strengthen team cohesion by providing an opportunity for teammates to socialise and bond [75]. This supports other findings of association between levels of alcohol consumption and team social cohesion [17] and the culture of alcohol consumption and sports club involvement in many countries [12, 13]. These findings, along with those of this study highlight the importance of clubs to provide opportunity for teammates to socialise together in order to strengthen team cohesion, in environments that support responsible alcohol consumption.

Despite the majority of respondents indicating that they would like more healthy food and beverage options available in their clubs, only a small number of clubs in this study sold mostly healthy options. Club representatives of community football clubs in Australia are in favour of providing a greater range of healthy food options in their club [24]. Increasing the availability and promoting healthy food and beverages in community football clubs has been found to increase sales of these products [67]. Intervention could involve encouraging young adults to eat healthier to complement their athletic and sporting goals. Such an intervention would need to address potential barriers including time restraints, financial implications and peer influences [76]. Future interventions need to address potential barriers for selling healthy foods in sports canteens, which include cost, perceived lack of demand, perishability of healthy foods, storage requirements and lack of knowledge of canteen staff [24].

The significant association between alcohol-related sponsorship and alcohol consumption in sport players provides further evidence to support replacement alcohol sponsorship in sports. There have been calls for government regulation of alcohol sponsorship in sport in Australia, with community members supporting the replacement of alcohol sponsorship in community sport clubs if compensated for lost revenue [50, 77]. Banning of tobacco sponsorship in sport provides examples of effective strategies that could be applied to alcohol sponsorship [78].

Policy development can assist sports clubs in implementing health promotion initiatives; however, it is important for clubs to receive support from health agencies, which can include training or advice and sample policies [72]. Capacity building [79] and organisational change [80] are also important factors in helping clubs adopt and implement health promotion strategies.

The findings of this study highlight the associations between club connectedness, team cohesion and mental wellness. Young adults were active volunteers, further enhancing their connection to club. Participants wanted healthy food and beverage options available at their clubs. However, connectedness and team cohesion were associated with higher levels of alcohol consumption and related harms. Given the role of alcohol in Australian sporting culture [11] this is not surprising. Health promotion intervention should harness the enthusiasm of young adults involved with their sports clubs. Based 
on the findings of this study key strategies for healthier clubs include:

Provide acceptable alternatives to high levels of alcohol consumption to enhance team cohesiveness and club connectedness;

Replace alcohol sponsorship (ensuring clubs do not lose revenue);

Capitalise on young adults' desire for healthier food and beverage options; and

Support and promote volunteer opportunities within clubs.

\section{Limitations}

Females were more likely to respond to the survey, however, other studies of similar age groups have also reported a majority of female respondents [35, 42, 81]. The small sample is a limitation of this study. Recent data suggests $68 \%$ of Australian 18-24 year olds participated in sport-related activity in the last 12 months [82]. Budget and time constraints influenced recruitment. Recruitment relied on self-selection, which may bias findings. The data collection was conducted during the winter months with the majority of respondents playing winter sports, and the majority from the Perth metropolitan area. Consequently, the sample may not be representative of the community sporting population aged 18-30 years within Western Australia. A higher proportion of male participants, and greater representation from rural sports clubs would strengthen the study. Future research could involve conducting the survey during the summer months, which would allow for a comparison by seasons, promoting the survey in rural and regional areas of Western Australia and including more structured recruitment procedures.

\section{Conclusion}

Previous studies have highlighted the association between high levels of alcohol consumption and participation in community sports clubs; however, there has been little focus on the association between connectedness, alcohol consumption and other health enhancing behaviours such as availability of healthy food and beverage options. The findings of this study highlight the benefits of club connectedness in creating a community and providing a sense of belonging and connection. The association between club connectedness, team cohesion, in particular team social cohesion, and higher levels of alcohol consumption, highlights the need for tailored interventions to focus on enhancing responsible alcohol consumption while maintaining club connectedness and team cohesion. The study findings also highlight the importance of attracting and retaining volunteers, especially young adults. The opportunity for sports clubs to promote other health enhancing behaviours, particularly associated with food and beverage consumption was also evident. It is unrealistic to expect clubs to be able to navigate the changes required and implement comprehensive health promotion programs without both structural and educative support.

\section{Abbreviations}

AUDIT-C: Alcohol Use Disorders Identification Test-Consumption; BCEOA: Brief Comprehensive Effects of Alcohol Scale; WEMWBS: WarwickEdinburgh Mental Well-being Scale; GEQ: Group Environment Questionnaire; AFL: Australian Football League

\section{Acknowledgements}

Not applicable.

\section{Authors' contributions}

$\mathrm{SB}, \mathrm{BM}, \mathrm{J}$ J and LP conceptualised the initial study. SB and ME developed initial drafts of the paper. ME collected data. ME and SB analysed data. All authors read and approved the final manuscript.

\section{Funding}

This research was funded by Healthway through an Australian Health Promotion Association (AHPA) Health Promotion Scholarship that was awarded to M.E.

\section{Availability of data and materials}

The datasets used and/or analysed during the current study are available from the first author on reasonable request.

\section{Ethics approval and consent to participate}

Ethics approval was obtained from the Curtin University Human Research Ethics Committee (HRE2016-0241). Participants provided written online consent.

\section{Consent for publication \\ Not applicable.}

\section{Competing interests}

No authors of this paper have competing interests.

Received: 30 September 2019 Accepted: 3 May 2020

Published online: 19 May 2020

References

1. Australian Bureau of Statistics. National health survey: first results, Australia 2017-18. Canberra: ABS; 2018.

2. Geidne S, Quennerstedt M, Eriksson C. The youth sports club as a healthpromoting setting: an integrative review of research. Scand J Public Health. 2013;41(3):269-83.

3. Sabo D, Veliz P. Go out and play: youth sports in America. East Meadow: Women's Sports Foundation; 2008.

4. Commonwealth of Australia. National sport and active recreation policy framework. Canberra: Commonwealth of Australia; 2011.

5. Fraser-Thomas JL, Côté J, Deakin J. Youth sport programs: an avenue to foster positive youth development. Phys Educ Sport Pedagog. 2005;10(1): 19-40.

6. Druglnfo Clearinghouse. Prevention research quarterly: alcohol and community sporting clubs. Melbourne: Australia Druglnfo Clearinghouse; 2009 .

7. Blomfield C, Barber B. Australian adolescents' extracurricular activity participation and positive development: is the relationship mediated by peer attributes? Aust J Educ Dev Psychol. 2010;10:114-28.

8. Fredricks JA, Eccles JS. Is extracurricular participation associated with beneficial outcomes? Concurrent and longitudinal relations. Dev Psychol. 2006;42(4):698.

9. Norton BL. Does community connectedness matter?: exploring the association between protective social factors and preventive health behaviors in a culturally diverse, environmentally dtressed context. Doctoral dissertation. Oklahoma: University of Oklahoma; 2006. 
10. Brown KM, Hoye R, Nicholson M. Self-esteem, self-efficacy, and social connectedness as mediators of the relationship between volunteering and well-being. J Soc Serv Res. 2012;38(4):468-83.

11. Kingsland M, Wolfenden L, Tindall J, Rowland B, Lecathelinais C, Gillham K, et al. Tackling risky alcohol consumption in sport: a cluster randomised controlled trial of an alcohol management intervention with community football clubs. J Epidemiol Community Health. 2015;69(10):993-9.

12. Lindsay J, Kelly P, Harrison L, Hickey C, Advocat J, Cormack S. 'What a great night': the cultural drivers of drinking practices among 14-24 year-old Australians. Melbourne: Monash University, Deakin University, Drinkwise, and the Australian Government Department of Health and Ageing; 2009.

13. Rowland B, Tindall J, Wolfenden L, Gillham K, Ramsden R, Wiggers J. Alcohol management practices in community football clubs: association with risky drinking at the club and overall hazardous alcohol consumption. Drug Alcohol Rev. 2015;34:438-46.

14. Grossbard J, Hummer J, LaBrie J, Pederson E, Neighbors C. Is substance use a team sport? Attraction to team, perceived norms, and alcohol and marijuana use among male and female intercollegiate athletes. J Appl Sport Psychol. 2009;21(3):247-61.

15. O'Brien KS, Kolt GS, Webber A, Hunter JA. Alcohol consumption in sport: the influence of sporting idols, friends and normative drinking practices. Drug Alcohol Rev. 2010;29(6):676-83.

16. Zhou J, O'Brien KS, Heim D. Alcohol consumption in sportspeople: the role of social cohesion, identity and happiness. Int Rev Sociol Sport. 2014;49(34):278-93.

17. Zamboanga BL, Rodriguez L, Horton NJ. Athletic involvement and its relevance to hazardous alcohol use and drinking game participation in female college athletes: a preliminary investigation. J Am Coll Heal. 2008; 56(6):651-6

18. Spink KS, Wilson KS, Odnokon P. Examining the relationship between cohesion and return to team in elite athletes. Psychol Sport Exerc. 2010;11(1):6-11.

19. Roche AM, Bywood P, Borlagdan J, Lunnay B, Freeman T, Lawton L, et al, Young people and alcohol: the role of cultural influences in. National Research Centre for Education and Training on Addiction: Adelaide; 2007.

20. Morris L, Sallybanks J, Willis K, Makkai T. Sport, physical activity and antisocial behaviour in youth. Youth Stud Aust. 2004;23(1):47.

21. Hickey C, Cormack S, Kelly P, Lindsay J, Harrison L. Sporting clubs, alcohol and young people: enduring tensions and emerging possibilities. ACHPER Aust Healthy Lifestyles J. 2009;56(1):17-21.

22. Kelly B, Chapman K, King L, Hardy L, Farrell L. Double standards for community sports: promoting active lifestyles but unhealthy diets. Health Promot J Aust. 2008;19(3):226-8.

23. Carter M, Signal LN, Edwards R, Hoel J. Competing teammates: food in New Zealand sports settings. Health Promot Int. 2019;34(4):803-10.

24. Young K, Kennedy V, Kingsland M, Sawyer A, Rowland B, Wiggers J, et al. Healthy food and beverages in senior community football club canteens in New South Wales, Australia. Health Promot J Aust. 2012;23(2):149-52.

25. Australian Bureau of Statistics. Involvement in organised sport and physical activity, Australia, April 2010. Canberra: ABS; 2010.

26. Rowland BC, Wolfenden L, Gillham K, Kingsland M, Richardson B, Wiggers J. Is alcohol and community sport a good mix? Alcohol management, consumption and social capital in community sports clubs. Aust N Z J Public Health. 2015;39:210-5.

27. Hildebrand J, Burns S, Zhao Y, Lobo R, Howat P, Allsop S, et al. Potential and challenges in collecting social and behavioral data on adolescent alcohol norms: comparing respondent-driven sampling and web-based respondentdriven sampling. J Med Internet Res. 2015;17(12):e285.

28. Saunders JB, Aasland OG, Babor TF, De la Fuente JR, Grant M. Development of the alcohol use disorders identification test (AUDIT): WHO collaborative project on early detection of persons with harmful alcohol consumption-II. Addiction. 1993;88(6):791-804.

29. Hallett J, Howat PM, Maycock BR, McManus A, Kypri K, Dhaliwal SS. Undergraduate student drinking and related harms at an Australian university: web-based survey of a large random sample. BMC Public Health. 2012;12(1):37.

30. Kypri K, Paschall MJ, Langley J, Baxter J, Cashell-Smith M, Bourdeau B. Drinking and alcohol-related harm among new Zealand university students: findings from a national web-based survey. Alcohol Clin Exp Res. 2009;33(2): 307-14

31. Bush K, Kivlahan DR, McDonell MB, Finn S, Bradley K. The AUDIT alcohol consumption questions (AUDIT-C): an effective brief screening test for problem drinking. Arch Intern Med. 1998;158:1789-95.
32. Zhou J, Heim D, O'Brien K. Alcohol consumption, athlete identity and happiness among student sportspeople as a function-type. Alcohol Alcohol. 2015;50(6):617-23.

33. Kokotailo PK, Egan J, Gangnon R, Brown D, Mundt M, Fleming M. Validity of the alcohol use disorders identification test in college students. Alcohol Clin Exp Res. 2004;28:914-20.

34. Hart E, Burns $\mathrm{S}$. The relationship between alcohol consumption and related harm among young university students. Health Promot J Aust. 2016;27(1):15-20.

35. Burns S, Jancey J, Crawford G, Hallett J, Portsmouth L, Longo J. A cross sectional evaluation of an alcohol intervention targeting young university students. BMC Public Health. 2016;16(1):610.

36. McGee R, Kypri K. Alcohol-related problems experienced by university students in New Zealand. Aust N Z J Public Health. 2004;28(4):321-3.

37. Wechsler H, Lee JE, Kuo M, Seibring M, Nelson TF, Lee $H$. Trends in college binge drinking during a period of increased prevention efforts: findings from 4 Harvard School of Public Health College alcohol study surveys: 1993-2001. J Am Coll Heal. 2002;50(5):203-17.

38. Ham LS, Stewart SH, Norton PJ, Hope DA. Psychometric assessment of the comprehensive effects of alcohol questionnaire: comparing a brief version to the original full scale. J Psychopathol Behav Assess. 2005;27(3):141-58.

39. Wang PZ. Assessing motivations for sports volunteerism. Adv Consum Res. 2004;31:420-5.

40. McNeely CA, Nonnemaker JM, Blum RW. Promoting school connectedness: evidence from the national longitudinal study of adolescent health. J Sch Health. 2002;72(4):138-46.

41. Lester L, Waters S, Cross D. The relationship between school connectedness and mental health during the transition to secondary school: a path analysis. J Psychol Couns Sch. 2013;23(2):157-71.

42. Burns SK, Maycock B, Hildebrand J, Zhao Y, Allsop S, Lobo R, et al. Development and testing of the youth alcohol norms survey (YANS) instrument to measure youth alcohol norms and psychosocial influences. BMJ Open. 2018:8(5):e019641.

43. Carron AV, Widmeyer WN, Brawley LR. The development of an instrument to assess cohesion in sport teams: the group environment questionnaire. $J$ Sport Psychol. 1985;7(3):244-66

44. Callow N, Smith MJ, Hardy L, Arthur CA, Hardy J. Measurement of transformational leadership and its relationship with team cohesion and performance level. J Appl Sport Psychol. 2009;21(4):395-412.

45. Lafferty ME, Wakefield C, Brown H. "We do it for the team"- studentathletes' initiation practices and their impact on group cohesion. Int J Sport Exerc Psychol. 2017;15(4):438-46.

46. Van Raalte JL, Cornelius AE, Linder DE, Brewer BW. The relationship between hazing and team cohesion. J Sport Behav. 2007:30(4):491-507.

47. Tennant R, Hiller L, Fishwick R, Platt S, Joseph S, Weich S, et al. The WarwickEdinburgh mental well-being scale (WEMWBS): development and UK validation. Health Qual Life Outcomes. 2007;5(1):63.

48. Nicholls AR, Levy AR, Carson F, Thompson MA, Perry JL. The applicability of self-regulation theories in sport: goal adjustment capacities, stress appraisals, coping, and well-being among athletes. Psychol Sport Exerc. 2016;27:47-55.

49. Zhou J, Heim D, Levy A. Sports participation and alcohol use: associations with sports-related identities and well-being. J Stud Alcohol Drugs. 2016;77(1):170-9.

50. Victorian Health Promotion Foundation. Community attitudes survey: healthy community sporting environments; 2010.

51. O'Brien KS, Kypri K. Alcohol industry sponsorship and hazardous drinking among sportspeople. Addiction. 2008;103(12):1961-6.

52. O'Brien KS, Miller PG, Kolt GS, Martens MP, Webber A. Alcohol industry and non-alcohol industry sponsorship of sportspeople and drinking. Alcohol Alcohol. 2011;46(2):210-3.

53. Bryman A. Social research methods. New York: Oxford University Press; 2004

54. Dancey CP, Reidy J. Statistics without Maths for psychology, Pearson education. 5th ed: 2011.

55. O'Brien KS, Ali A, Cotter JD, O'Shea RP, Stannard S. Hazardous drinking in New Zealand sportspeople: level of sporting participation and drinking motives. Alcohol Alcohol. 2007;42(4):376-82

56. O'Farrell A, Kingsland M, Kenny S, Eldin N, Wiggers J, Wolfenden L, et al. A multi-faceted intervention to reduce alcohol misuse and harm amongst sports people in Ireland: a controlled trial. Drug Alcohol Rev. 2018:37(1):14-22.

57. Tomon JE, Ting SR. Effects of team climate on substance use behaviors, perceptions, and attitudes of student-athletes at a large, public university. J Coll Stud Dev. 2010;51(2):162-79. 
58. Eime RM, Harvey JT, Brown WJ, Payne WR. Does sports club participation contribute to health-related quality of life? Med Sci Sports Exerc. 2010;42(5): $1022-8$.

59. Eime RM, Young JA, Harvey JT, Charity MJ, Payne WR. A systematic review of the psychological and social benefits of participation in sport for adults: informing development of a conceptual model of health through sport. Int J Behav Nutr Phys Act. 2013;10(1):135.

60. Armstrong S, Oomen-Early J. Social connectedness, self-esteem, and depression symptomatology among collegiate athletes versus nonathletes. J Am Coll Heal. 2009;57(5):521-6.

61. Schlesinger T, Egli B, Nagel S. 'Continue or terminate?' Determinants of longterm volunteering in sports clubs. Eur Sport Manag Q. 2013;13(1):32-53.

62. Australian Bureau of Statistics. Volunteers in sport, Australia, 2010. Canberra: ABS; 2010.

63. Cuskelly G, Hoye R, Auld C. Working with volunteers in sport New York: Routledge; 2006

64. Gray E, Khoo S-E, Reimondos A. Participation in different types of volunteering at young, middle and older adulthood. J Popul Res. 2012;29(4): 373-98.

65. Schlesinger T, Nagel S. Individual and contextual determinants of stable volunteering in sport clubs. Int Rev Sociol Sport. 2018;53(1):101-21.

66. Carter M, Edwards R, Signal L, Hoek J. Availability and marketing of food and beverages to children through sports settings: a systematic review. Public Health Nutr. 2012;15:1373-9.

67. Wolfenden L, Kingsland M, Rowland BC, Dodds P, Gillham K, Yoong SL, et al. Improving availability, promotion and purchase of fruit and vegetable and non sugar-sweetened drink products at community sporting clubs: a randomised trial. Int J Behav Nutr Phys Act. 2015;12:1.

68. Grunseit AC, MacNiven R, Orr R, Grassmayr M, Kelly B, Davies D, et al. Australian athletes' health behaviours and perceptions of role modelling and marketing of unhealthy products. Health Promotion J Aust. 2012;23(1):63-9.

69. Munt A, Partridge $S$, Allman-Farinelli M. The barriers and enablers of healthy eating among young adults: a missing piece of the obesity puzzle: a scoping review. Obes Rev. 2017;18(1):1-17.

70. O'Brien KS, Ferris J, Greenlees I, Jowett S, Rhind D, Cook PA, et al. Alcohol industry sponsorship and hazardous drinking in UK university students who play sport. Addiction. 2014;109(10):1647-54.

71. Brown K. Association between alcohol sports sponsorship and consumption: a systematic review. Alcohol Alcohol. 2016;51(6):747-55.

72. Dobbinson SJ, Hayman JA, Livingston PM. Prevalence of health promotion policies in sports clubs in Victoria, Australia. Health Promot Int. 2006;21(2): $121-9$.

73. Kokko S. Sports clubs as settings for health promotion: fundamentals and an overview to research. Scand J Public Health. 2014:42(15 suppl):60-5.

74. Rowland B, Allen F, Toumbourou JW. Association of risky alcohol consumption and accreditation in the 'good sports' alcohol management programme. J Epidemiol Community Health. 2012;66:684-90.

75. Zhou J, Heim D. A qualitative exploration of alcohol use among student sportspeople: a social identity perspective. Eur J Soc Psychol. 2016;46(5): 581-94.

76. Ashton LM, Hutchesson MJ, Rollo ME, Morgan PJ, Collins CE. Motivators and barriers to engaging in healthy eating and physical activity: a cross-sectional survey in young adult men. Am J Mens Health. 2017;11(2):330-43.

77. McCusker Centre for Action on Alcohol and Youth. It's not fair play: why alcohol must leave sport. Perth: Commonwealth of Australia 2017.

78. Kypri K, O'Brien K, Miller P. Time for precautionary action on alcohol industry funding of sporting bodies. Addiction. 2009;104(12):1949-50.

79. Casey MM, Payne WR, Eime RM, Brown SJ. Sustaining health promotion programs within sport and recreation organisations. J Sci Med Sport. 2009, 12(1):113-8.

80. Heward S, Hutchins C, Keleher H. Organizational change — key to capacity building and effective health promotion. Health Promot Int. 2007;22(2):170-8.

81. Hunt K, Burns S. Is there an association between social connectedness, social identity, alcohol consumption and mental health among young university students? Open J Prev Med. 2017;7(06):99-114.

82. Australian Sports Commission. AusPlay: participation data for the sports sector. Canberra: Commonwealth of Australia 2016.

\section{Publisher's Note}

Springer Nature remains neutral with regard to jurisdictional claims in published maps and institutional affiliations.

\section{Ready to submit your research? Choose BMC and benefit from}

- fast, convenient online submission

- thorough peer review by experienced researchers in your field

- rapid publication on acceptance

- support for research data, including large and complex data types

- gold Open Access which fosters wider collaboration and increased citations

- maximum visibility for your research: over $100 \mathrm{M}$ website views per year

At BMC, research is always in progress.

Learn more biomedcentral.com/submissions 